\title{
Rigorous predictions for prompt neutrino fluxes in view of VLV $v T$ upgrades
}

\author{
M.V. Garzelli, ${ }^{a, *}$ S.-O. Moch $^{a}$ and G. Sigl ${ }^{a}$ \\ ${ }^{a}$ II Institut für Theretische Physik, Universität Hamburg, \\ Luruper Chaussee 149, D-22761, Hamburg, Germany \\ E-mail: maria.vittoria.garzelli@desy.de, sven-olaf.moch@desy.de, \\ guenter.sigl@desy.de
}

The existence of a flux of prompt atmospheric neutrinos from the decay of heavy hadrons resulting from the interactions of cosmic rays with the atmospheric nuclei is predicted by theory. Very Large Volume Neutrino Telescopes, like Icecube, KM3NeT and Baikal-GVD, should be sensitive to this neutrino component, that represents a background for the neutrinos from far astrophysical sources. However, no clear experimental evidence of prompt neutrino fluxes has been found, at least so far. In particular, the prompt neutrino component well fits to zero even in the most recent analysis of High Energy Starting Events by the IceCube collaboration, published last autumn. On the other hand, the analysis of through-going muon tracks, more sensitive to prompt neutrinos than the previous one, has established an upper limit on prompt neutrino fluxes. Our collaboration has been active in providing accurate predictions for prompt neutrino fluxes in the last few years, on the basis of rigorous QCD calculations, and in assessing many of the uncertainties related to these predictions. We discuss our most recent results and their uncertainties, which we believe constitute the most accurate and comprehensive prediction of prompt neutrino fluxes available at present, and show how they challenge the present experimental limits. We are confident that, increasing the experimental capabilities and statistical sample, as possible through e.g. the IceCube-Gen2 upgrade, will help in sharing further light on the prompt neutrino issues.

$37^{\text {th }}$ International Cosmic Ray Conference (ICRC 2021)

July 12 th - 23rd, 2021

Online - Berlin, Germany

\footnotetext{
*Presenter
} 


\section{Introduction}

A diffuse flux of neutrinos arises from the decays of hadrons produced by the interactions of cosmic rays (CR), impinging in the air atmosphere, with the atmospheric nuclei. If their energy is high enough, charged pions and kaons, abundantly produced in this kind of interactions, do not decay within the limited size of the atmospheric mantle of the Earth. Only heavy-flavoured hadrons, decaying much more promptly but having smaller production cross-sections, are still able to produce a sizable flux of neutrinos. This leads to a non-trivial atmospheric neutrino flux energy spectrum, dominated by different components in different regions, i.e. the conventional component at low energy and the prompt component at high energy, involving a change of slope. The precise energy range where the two components are approximately the same and the transition from the dominance of one to that of the other occurs, is characterized by uncertainties, which follow from our present uncertainties on both the conventional and the prompt fluxes.

The diffuse atmospheric neutrino flux is a relevant background for accurate estimates of the diffuse astrophysical neutrino flux carried out at Very Large Volume Neutrino Telescopes (VLV $v$ Ts). The astrophysical neutrino flux is generated by neutrinos from far astrophysical sources. Its precise knowledge/accurate measurement is important for the astroparticle physics community because it may allow to shed light on open questions such as the dominant interaction mechanisms in the vicinity of these sources and the origin of CRs. It is not possible to answer these questions by looking exclusively to CRs due to their charge, that does not allow to track back their trajectory from the Earth to their origin. On the other hand, high-energy neutrinos reaching the Earth, in absence of electroweak bosons on their path, point back to their galactic and/or extragalactic sources.

IceCube has detected neutrino events up to energies of some PeV. After 2635 days of data taking, the most recent High-Energy Starting Event (HESE) analysis [1] reported a few neutrino events with energies $E_{v, \mathrm{LAB}}>3 \cdot 10^{5} \mathrm{GeV}$. In this energy regime the conventional neutrino flux is expected to start being suppressed, until the transition conventional/prompt occurs, leading to an atmospheric neutrino flux high-energy tail dominated by the prompt component. On the other hand, the largest number of detected events comes from the region $E_{v, \mathrm{LAB}} \sim 10^{5} \mathrm{GeV}$, where the conventional component is expected to be very sizable. On the basis of a simultaneous fit of the atmospheric and astrophysical components, using the 60 events with $E_{v, \mathrm{LAB}}>60 \mathrm{TeV}$, the IceCube collaboration came to the conclusion that the astrophysical flux is compatible with a single power-law spectrum with spectral index $\Gamma=-2.87_{-0.19}^{+0.20}$, whereas the prompt neutrino flux, for which they fix the shape and vary the normalization, is well compatible with zero. On the other hand, as shown in the following, current up-to-date theoretical predictions provide an expected non-null flux of prompt neutrinos, although with large uncertainties. In this work we discuss the present status of these uncertainties, and two possible explanations of the fact that the flux seen by IceCube so far is instead null: cold nuclear matter effects, which have been accounted for only in a rough way in presented predictions, and low-statistics issues.

\section{Present uncertainties on prompt neutrino fluxes}

Atmospheric neutrino fluxes reaching the Earth's surface can be computed by solving a system of coupled differential equations related to the variation, with atmospheric depth, of the fluxes of 
different kinds of particles and hadrons. These equations include interaction, decay and regeneration terms, capable of modifying the flux of each species. Crucial elements entering these terms are the energetic spectra of daughter particles generated by parent particles through the aforementioned processes, weighted with the parent energy spectra and integrated over the range of energies of the parent. It follows that prompt neutrinos of energies $E_{v, \mathrm{LAB}} \sim O(\mathrm{PeV})$ arise from the decay of heavy hadrons produced in collisions at larger energies. Due to the shape of the integrand functions, most of them are actually produced in collisions at center-of-mass energies of the order of the Large Hadron Collider (LHC) ones. Data have been collected at the LHC concerning the production of heavy hadrons. In particular, the LHCb experiment has reported double-differential cross-sections as a function of the transverse momentum and rapidity in various rapidity bins in the interval $2<y<4.5$ for the single-inclusive production of various heavy-hadrons, at various center-of-mass energies. These data represent a good benchmark for QCD calculations. Although prompt neutrinos are produced by interactions on an even wider rapidity range, it has become common practice of the groups aiming at computing prompt neutrino fluxes using $\mathrm{QCD}$, to compare their predictions to the LHC available experimental data. Other experiments, in particular ATLAS and ALICE, have collected data at smaller rapidities, which can also be considered for theory/data comparisons.

It is possible to go beyond the simple level of data/theory comparison, by using the available data to fit some of the non-perturbative input entering the QCD calculations. This is e.g. the path undertaken by the PROSA collaboration. All present LHCb data on single-inclusive charmed hadron production plus selected $\mathrm{LHCb}$ data on single-inclusive bottomed meson production at a center-of-mass energy $\sqrt{s}=7 \mathrm{TeV}$ plus recent ALICE data, have been used to fit the PROSA parton distribution functions (PDFs), in particular the gluon and the sea quark PDFs in longitudinal momentum fraction $(x)$ regions not covered by the HERA deep inelastic scattering data, also included in the fit and representing the core of all modern PDF fits. In fact, heavy-flavour production at the LHC is sensitive to PDFs in the $x$ regions $10^{-6}<x<10^{-4}$ and $x>10^{-1}$ [2], where the current constraints from other experiments are very weak. This has led to a new fit, the PROSA 2019 PDF set [3], with reduced uncertainties on the gluon and sea quark PDFs with respect to the past. A similar analysis, although limited to the LHCb charm data only, has been performed indipendently by another collaboration. Their latter work [4], based on the reweighting of an already existing PDF set with the LHC charm production data, as led to NLO PDFs well compatible, within uncertainty bands, with those obtained by the PROSA collaboration.

We have used the PROSA 2019 PDF sets in the fixed-flavour and variable-flavour number schemes (FFNS and VFNS), as input for the computation of prompt neutrino fluxes, working in QCD in collinear factorization, with different approaches, including the charm finite-mass effects in different ways. In the collinear framework, the cross-section for single inclusive heavy hadron production can be factorized in terms of PDFs, partonic cross-sections and fragmentation. The latter step has been described by two alternative methods, either a fragmentation function (+ fragmentation fraction), or a parton shower + hadronization approach, finding results consistent among each other. The partonic cross-section has been computed in perturbative QCD (pQCD) including radiative corrections up to next-to-leading order (NLO), with finite charm-mass effects. The $K$-factor is large [5], and the shape of differential distributions turns out to change when increasing the perturbative order of the computation from leading order (LO) to NLO. This is a sign that working at LO leads to a very poor approximation for the heavy-quark production 

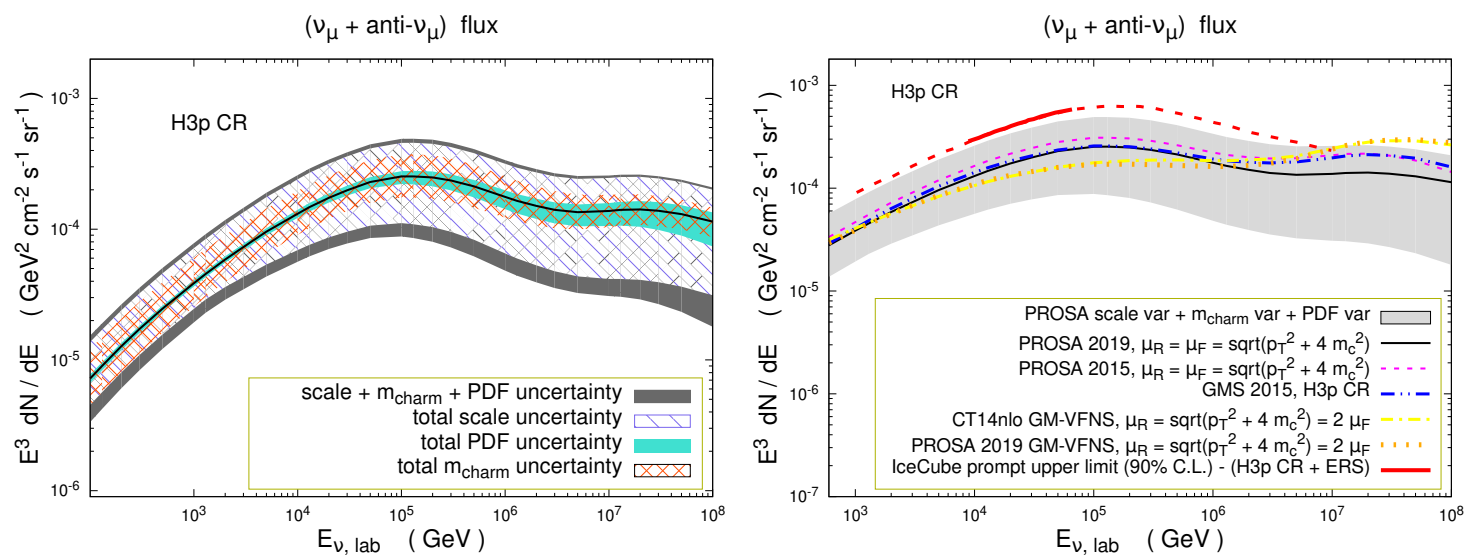

Figure 1: Left: prompt $\left(v_{\mu}+\bar{v}_{\mu}\right)$ fluxes and their QCD uncertainties related to renormalization and factorization scale, PDF and charm mass variation [3]. Hard-scattering matrix-elements include radiative corrections up to NLO. The NLO PROSA 2019 FFNS PDF set has been used as input, with a charm mass value $m_{c}=1.43 \mathrm{GeV}$. The $\mathrm{H} 3 \mathrm{p}$ all-nucleon primary $\mathrm{CR}$ spectrum is also an input of the computation. Right: comparison of the previous prediction with other predictions in the literature [5-7] and with the IceCube upper limit on prompt $\left(v_{\mu}+\bar{v}_{\mu}\right)$ fluxes from Ref. [8]. See text for more detail.

cross-sections. Even NLO cross-sections are still prone to large uncertainties, hinting to a slowly converging pQCD series. Going to higher-orders is fundamental to reduce the uncertainties.

Our predictions for prompt $\left(v_{\mu}+\bar{v}_{\mu}\right)$ fluxes from charm production and decay are presented in Fig. 1, using as input the H3a all-nucleon CR primary spectrum. Together with central predictions, obtained with the PROSA 2019 FFNS PDF set and a NLO calculation matched to parton shower according to the POWHEG method, and complemented by hadronization and hadron decay effects, various QCD uncertainties are shown in the left panel. Those related to renormalization and factorization scale variation are indeed the biggest. Uncertainties related to PDF variation, computed using as input all members of the PROSA PDF set, are much smaller, at least up to $E_{v, \mathrm{LAB}}$ values of the order of the maximum probed by IceCube so far. PDF uncertainties increase with $E_{v, \mathrm{LAB}}$, considering that the most energetic neutrinos are indeed produced by the collisions at the highest center-of-mass energy, corresponding to the more extreme $x$ values, where the PDFs are less constrained. Uncertainties related to our inaccurate knowledge of the charm mass value decrease with $E_{v}$, because charm mass effects are comparably more important at lower center-of-mass energies than at higher ones. Our calculation refer to charm masses renormalized in the on-shell scheme. It is worth noticing that the PROSA 2019 PDF set was extracted together with heavy-quark mass values renormalized in the $\overline{\mathrm{MS}}$ scheme, preserving correlations. The conversion of mass values from the $\overline{\mathrm{MS}}$ to the on-shell scheme causes an increase of mass uncertainties, related to the renormalon ambiguity $\sim O\left(\Lambda_{\mathrm{QCD}}\right)$. This is an intrinsic uncertainty accompanying all heavy-quark masses in the on-shell scheme, related to the fact that quarks are confined instead of being free asymptotic states, so the concept of pole mass is ill defined for these particles (for more detail and discussions on the uncertainties of predictions with charm masses in different renormalization schemes see e.g. [9]). 


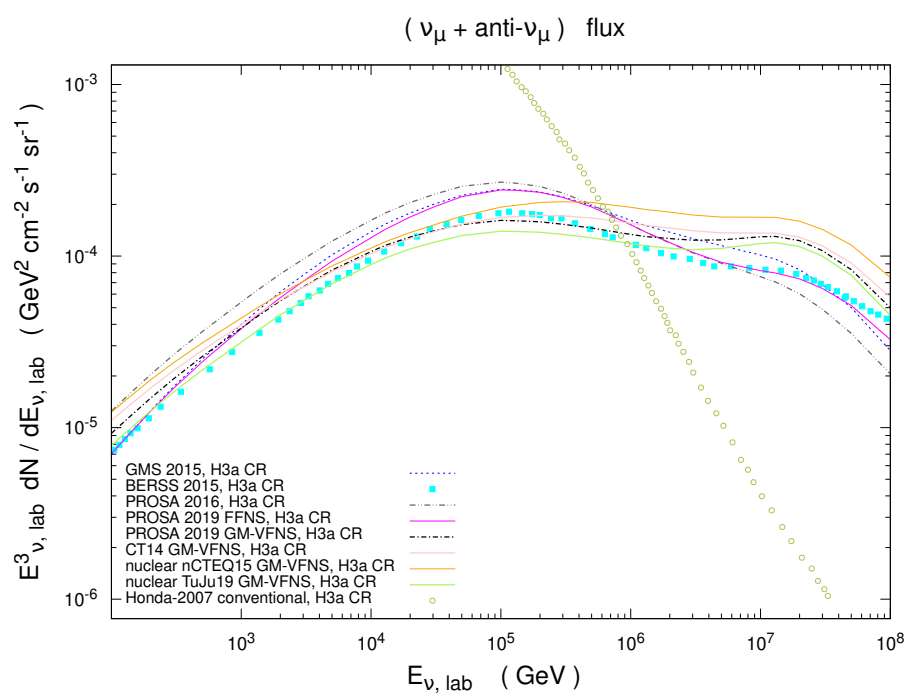

Figure 2: Central predictions for prompt $\left(v_{\mu}+\bar{v}_{\mu}\right)$ fluxes using different approaches, adopting as input various proton and nuclear PDFs and charm mass values. Besides predictions shown here for the first time, we also present those of Ref. [3, 5, 10]. Predictions for conventional fluxes by Ref. [11], reweighted to the H3a CR primary all-nucleon spectrum, are also shown.

The HESE analysis mentioned in the Introduction does not have an upper limit on the maximum energy of neutrinos. However, due to low statistics at high-energy, the majority of HESE events comes from an energy region where the conventional neutrino component is large. Therefore the HESE analysis is not particularly sensitive to the prompt component. Upper limits on the latter were instead established in a dedicated analysis of through-going muon tracks from the Northest Hemisphere [8]. Our theoretical predictions, including their uncertainties, are still within these limits, as shown in the right panel of Fig. 1. It is expected that these limits will become stronger in future $\operatorname{VLV} v \mathrm{~T}$ analyses with increased statistics, challenging the theoretical predictions.

In Fig. 2, we collect a series of predictions for prompt neutrino fluxes obtained with different calculations and different PDFs/charm mass values. All predictions use as input the H3a CR primary all-nucleon flux and all calculations have been performed in collinear factorization and include hard-scattering matrix-elements at NLO. The total accuracy of these predictions differs, however, depending on the method used to do the calculation. In particular, the BERSS 2015 flux [10] has fixed-order NLO accuracy, the GMS 2015 [5] and PROSA 2019 FFNS predictions [3] have NLO + Shower Monte Carlo accuracy, the GM-VFNS predictions, which effectively resums logarithms of $\left(p_{T} / m_{c}\right)$ for large transverse momentum of the produced charm quark/D-meson, have NLO + Next-to-leading-logarithmic (NLL) accuracy. For the latter approach, we present predictions for proton-Nitrogen $(\mathrm{N})$ collisions in two different approximation: a) the superposition model, according to which the Nitrogen nucleus is decomposed into 7 proton and 7 neutrons, also used in all other approaches, b) a cold nuclear matter model, according to which all nuclear effects in proton-nucleus collisions are supposed to be reabsorbed in nuclear PDFs. This assumption, although offering a very simplified picture not catching the distinctive features of multiple cold 

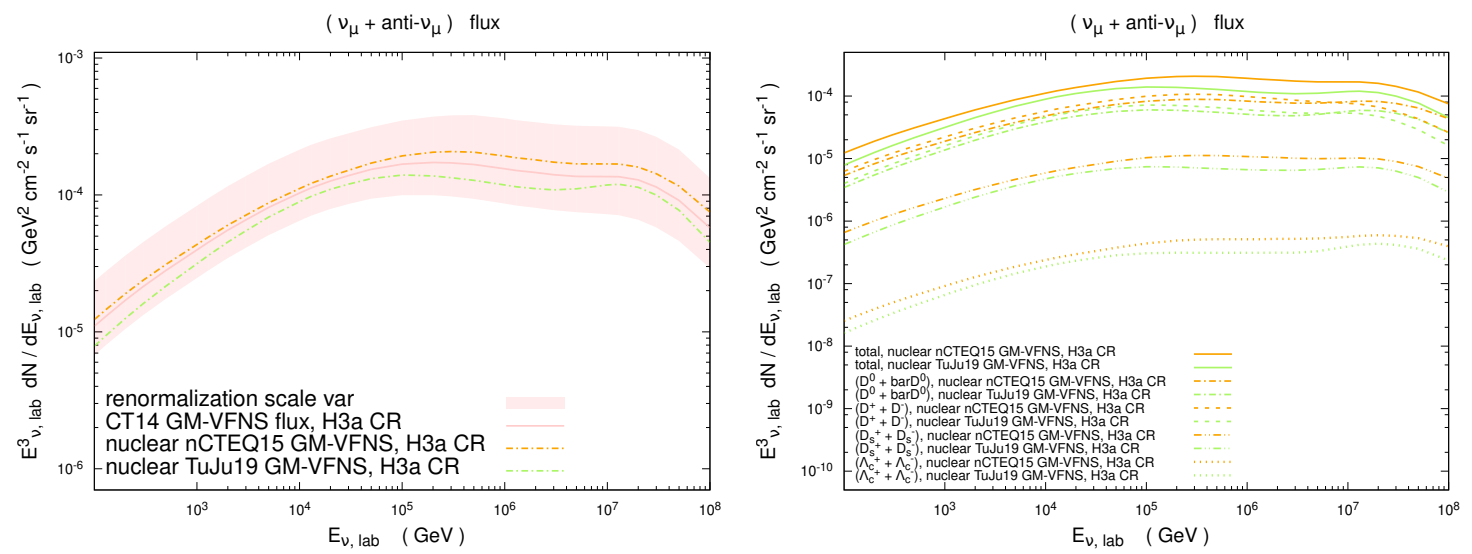

Figure 3: Left: Prompt $\left(v_{\mu}+\bar{v}_{\mu}\right)$ fluxes computed using the GM-VFNS nucleon-nucleon approach with the PROSA 2019 PDF set and the superposition model (pink), together with their renormalization scale uncertainties, compared to those obtained in a GM-VFNS nucleon-Nucleus approach with proton and Nitrogen PDFs from the nCTEQ15 (orange) and the TuJu19 analyses (green). Right: contributions to the predictions in the GM-VFNS nucleon-Nucleus approach of the left panel, arising from $D^{0}+\bar{D}^{0}, D^{+}+D^{-}$, $D_{s}^{+}+D_{s}^{-}$and $\Lambda_{c}^{+}+\Lambda_{c}^{-}$production and decay.
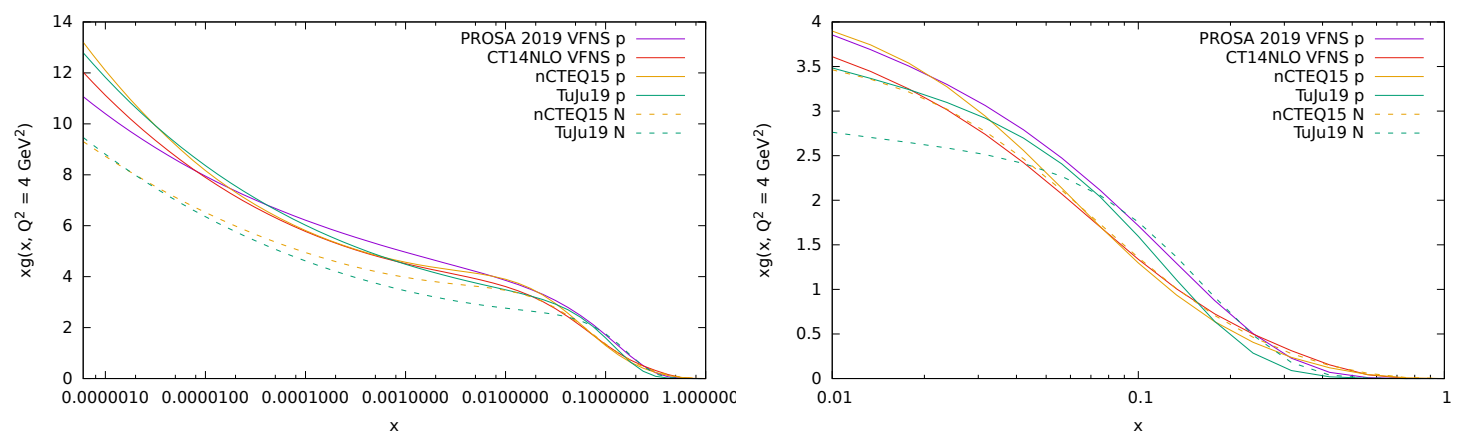

Figure 4: The gluon distribution in different proton and nuclear PDF fits, as a function of the longitudinal momentum fraction $x$. The right panel zooms over the large- $x$ region.

nuclear matter effects which might actually play a role, is currently used in nuclear PDF fits as well. For case a) we consider the case in which the proton and neutron PDF are described by the CT14nlo PDF set, that we already presented in Ref. [7], and the case where they are described by the PROSA VFNS PDF set [3]. We compare the predictions to the conventional neutrino flux computed by Honda [11] and reweighted to the H3a CR spectrum as well. For the case b) we consider the proton and nitrogen PDFs from the nCTEQ15 PDF analysis [12] and those from the TuJu19 analysis [13]. The transition region is around $\sim 10^{6} \mathrm{GeV}$. We observe that the various central predictions are compatible among each other within a factor of three, with differences increasing at increasing $E_{v, \mathrm{LAB}}$. The exact location of the transition point depends on the uncertainty of the predictions.

In case of present nuclear PDF fits, predictions for prompt neutrino fluxes can be smaller or larger than in the superposition model, depending on the nuclear PDF sets used. For the PDF cases 
considered in this work, the differences between predictions computed according to approximations a) and b) turn out to lie within scale uncertainties, as shown in Fig. 3, left. At the energies of interest of present $\operatorname{VLV} v \mathrm{~T}$, the largest contribution to prompt neutrino fluxes come from the production and decay of $D^{0}+\bar{D}^{0}$ and $D^{+}+D^{-}$mesons, as shown in Fig. 3, right. The shape of the energy spectra is sensitive to the (nuclear) PDF used.

The results in Fig. 3 are the consequence of the PDF behaviour at low $Q^{2}$. We report the gluon distribution as a function of $x$ in Fig. 4, focusing both on a wide $x$ range, extending to low $x$ values, and on the interval of largest $x$ values. At small $x$ the considered nuclear PDFs are suppressed with respect to $p$ PDFs, but still compatible with the latter within the uncertainty bands. On the other hand, at large $x$, one can notice that no antishadowing is present for the $\mathrm{N}$ nucleus for the central PDFs that we considered, that resemble quite closely the $p$ ones.

\section{Conclusions}

Upgrades of IceCube to IceCube-Gen2 and additional results from KM3NeT and Baikal-GVD, complementing those of IceCube, will be fundamental to increase the statistics of neutrino events with $E_{v} \sim O(\mathrm{PeV})$ and help solving the prompt neutrino issue, i.e. the fact that, notwithstanding present theory predictions point to the existence of a prompt neutrino component, this has not been detected so far. Simultaneous better constraints on nuclear PDFs, in particular on the gluon, as possible through the use of heavy-flavour production data which will be collected during Run 3 and the High-Luminosity Phase of the LHC, both by standard experiments (in particular LHCb) in $p A$ runs, and by new experiments sensitive to forward neutrinos, complemented by future data at the electron-ion and Large hadron-electron colliders, will allow to better assess the role of cold nuclear matter effects in modifying the prompt neutrino flux, with respect to present predictions mostly based on $N N$ interactions and the superposition model.

In the meanwhile, further analyses of the prompt neutrino fluxes, in particular focusing on the $\left(v_{e}+\bar{v}_{e}\right)$ samples, for which the transition between the conventional and the prompt component is supposed to occur at lower energies than in the $\left(v_{\mu}+\bar{v}_{\mu}\right)$ case, and on dedicated $\left(v_{\tau}+\bar{v}_{\tau}\right)$ searches, are indeed welcome and might already allow to make progress on the prompt neutrino puzzle.

We recommend that in forthcoming analyses uncertainties related to the shape of the distributions of prompt neutrino fluxes, besides those related to the normalization, are included, and that the most rigorous predictions are considered by the experimental collaborations in this respect.

\section{Acknowledgments}

We are grateful to the members of the PROSA collaboration and to M. Benzke and M. Walt for having provided various inputs for the computations of prompt neutrino fluxes presented in this work. This work has been supported in part by the german Bundesministerium für Bildung und Forschung (BMBF).

\section{References}

[1] IceCube Collaboration, R. Abbasi et al., The IceCube high-energy starting event sample: Description and flux characterization with 7.5 years of data, arXiv: 2011.03545. 
[2] PROSA Collaboration, O. Zenaiev et al., Impact of heavy-flavour production cross sections measured by the LHCb experiment on parton distribution functions at low x, Eur. Phys. J. C75 (2015), no. 8 396, [arXiv: 1503.04581].

[3] PROSA Collaboration, O. Zenaiev, M. V. Garzelli, et al., Improved constraints on parton distributions using LHCb, ALICE and HERA heavy-flavour measurements and implications for the predictions for prompt atmospheric-neutrino fluxes, JHEP 04 (2020) 118, [arXiv: 1911.13164].

[4] V. Bertone, R. Gauld, and J. Rojo, Neutrino Telescopes as QCD Microscopes, JHEP 01 (2019) 217, [arXiv: 1808.02034].

[5] M. V. Garzelli, S. Moch, and G. Sigl, Lepton fluxes from atmospheric charm revisited, JHEP 10 (2015) 115, [arXiv: 1507.01570$].$

[6] PROSA Collaboration, M. V. Garzelli et al., Prompt neutrino fluxes in the atmosphere with PROSA parton distribution functions, JHEP 05 (2017) 004, [arXiv: 1611.03815].

[7] M. Benzke, M. V. Garzelli, B. Kniehl, G. Kramer, S. Moch, and G. Sigl, Prompt neutrinos from atmospheric charm in the general-mass variable-flavor-number scheme, JHEP 12 (2017) 021, [arXiv: 1705.10386].

[8] IceCube Collaboration, M. G. Aartsen et al., Observation and Characterization of a Cosmic Muon Neutrino Flux from the Northern Hemisphere using six years of IceCube data, Astrophys. J. 833 (2016), no. 1 3, [arXiv: 1607.08006$].$

[9] M. V. Garzelli, L. Kemmler, S. Moch, and O. Zenaiev, Heavy-flavor hadro-production with heavy-quark masses renormalized in the $\overline{\mathrm{MS}}$, MSR and on-shell schemes, JHEP 04 (2021) 043, [arXiv:2009.07763].

[10] A. Bhattacharya, R. Enberg, M. H. Reno, I. Sarcevic, and A. Stasto, Perturbative charm production and the prompt atmospheric neutrino flux in light of RHIC and LHC, JHEP 06 (2015) 110, [arXiv: 1502.01076].

[11] M. Honda, T. Kajita, K. Kasahara, S. Midorikawa, and T. Sanuki, Calculation of atmospheric neutrino flux using the interaction model calibrated with atmospheric muon data, Phys. Rev. D 75 (2007) 043006, [astro-ph/0611418].

[12] K. Kovarik et al., nCTEQ15 - Global analysis of nuclear parton distributions with uncertainties in the CTEQ framework, Phys. Rev. D 93 (2016), no. 8085037 , [arXiv: 1509.00792].

[13] M. Walt, I. Helenius, and W. Vogelsang, Open-source QCD analysis of nuclear parton distribution functions at NLO and NNLO, Phys. Rev. D 100 (2019), no. 9096015 , [arXiv: 1908.03355]. 